\title{
Adequate tissue acquisition rate of peroral cholangioscopy- guided forceps biopsy
}

\author{
Takumi Onoyama^, Yohei Takeda, Soichiro Kawata, Hiroki Kurumi, Hiroki Koda, Taro Yamashita, \\ Wataru Hamamoto, Yuri Sakamoto, Kazuya Matsumoto, Hajime Isomoto \\ Division of Gastroenterology and Nephrology, Department of Multidisciplinary Internal Medicine, Faculty of Medicine, Tottori University, Yonago, \\ Tottori, Japan \\ Contributions: (I) Conception and design: T Onoyama; (II) Administrative support: H Isomoto; (III) Provision of study materials or patients: T \\ Onoyama; (IV) Collection and assembly of data: T Onoyama, Y Takeda, S Kawata, H Kurumi, H Koda, T Yamashita, W Hamamoto, Y Sakamoto, K \\ Matsumoto; (V) Data analysis and interpretation: T Onoyama; (VI) Manuscript writing: All authors; (VII) Final approval of manuscript: All authors. \\ Correspondence to: Takumi Onoyama. Division of Gastroenterology and Nephrology, Department of Multidisciplinary Internal Medicine, Faculty of \\ Medicine, Tottori University, Nishicho 36-1, Yonago, Tottori, Japan. Email: golf4to@yahoo.co.jp.
}

Background: Peroral cholangioscopy (POCS)-guided forceps biopsy is a method for diagnosing indeterminate biliary strictures and for the preoperative identification of the exact perihilar and distal margins of biliary tract cancer (BTC). However, POCS-guided forceps biopsy may result in an insufficient amount of specimen at times. Therefore, we evaluated the adequate tissue acquisition rate and the factors affecting the adequate tissue acquisition of POCS-guided forceps biopsy for the biliary tract.

Methods: Patients who underwent POCS-guided forceps biopsy for biliary disease between September 2016 and October 2018 at our hospital were enrolled retrospectively. We evaluated the adequate tissue acquisition rate of POCS-guided forceps biopsy for the biliary lesion and that for non-stenotic bile duct. In addition, the factors affecting the adequate tissue acquisition rate of POCS-guided forceps biopsy were evaluated.

Results: We enrolled 47 patients with biliary disease and performed POCS-guided forceps biopsy for biliary lesion and POCS-guided forceps mapping biopsy for non-stenotic bile duct in 40 and 36 patients, respectively. The adequate tissue acquisition rates of POCS-guided forceps biopsy for biliary lesions and that for non-stenotic bile duct were $86.4 \%$, and $68.9 \%$, respectively. In the multivariate logistic regression analyses, age, and previous biliary stenting before POCS were factors affecting the adequate tissue acquisition rate of POCS-guided forceps biopsy for the biliary lesion. For non-stenotic bile duct, the location of the biliary lesion, endoscopic sphincterotomy (EST), and procedure time of POCS were factors affecting the adequate tissue acquisition rate of POCS-guided forceps mapping biopsy.

Conclusions: Previous biliary stenting was a factor affecting a low tissue acquisition rate of POCS-guided forceps biopsy for the biliary lesion. In the POCS-guided forceps mapping biopsy, the location of the biliary lesion, EST, and procedure time were factors affecting tissue acquisition rates.

Keywords: Peroral cholangioscopy (POCS); biopsy; tissue acquisition rate; biliary tract cancer (BTC); accuracy

Submitted Mar 21, 2020. Accepted for publication Jul 20, 2020.

doi: $10.21037 /$ atm-20-2738

View this article at: http://dx.doi.org/10.21037/atm-20-2738

^ORCID: 0000-0003-0310-0720. 


\section{Introduction}

Biliary tract cancer (BTC), including intrahepatic cholangiocarcinoma, extrahepatic cholangiocarcinoma, gallbladder carcinoma, and ampullary carcinoma, is a disease with a poor prognosis. The 5 -year survival rate and median survival time of BTC was $18.7 \%$ and 8.5 months, respectively (1). Although the early diagnosis of BTC might improve the prognosis of that, BTC is often diagnosed at an advanced stage and is often unresectable because it is difficult to differentiate between BTC and benign biliary diseases, such as primary sclerosing cholangitis, immunoglobulin G subclass 4 (IgG4)-associated sclerosing cholangitis, xanthogranulomatous cholecystitis, and Mirizzi syndrome $(2,3)$. It is important to distinguish BTC from benign biliary disease, as the treatment strategies and prognoses differ.

Endoscopic retrograde cholangiopancreatography (ERCP)-related tissue acquisition, such as bile aspiration cytology, biliary brush cytology, and forceps biopsy, is commonly used to diagnose indeterminate biliary lesions. Although the specificity of the pathological examination of ERCP-related tissue acquisition for indeterminate biliary strictures is almost $100 \%$, the sensitivity of that is unsatisfactory, with a range of $6-72 \%(4,5)$.

Recently, the utility of peroral cholangioscopy (POCS)guided forceps biopsy to diagnose indeterminate biliary lesions has been reported $(6,7)$. However, the sensitivity of POCS-guided forceps biopsy for indeterminate biliary stricture is unsatisfactory $(60.1 \%)(8)$.

BTC has a clinical feature of superficial intraductal spread, in which epithelium extends continuously from the main lesion (9). The frequency of superficial intraductal spread in cholangiocarcinoma is reported to be $14.6 \%$ (10). The presence of superficial intraductal spread is related to non-curative resection for BTC so that it is essential to identify the exact perihilar and distal margins of the preoperative BTC. The usefulness of POCS-guided forceps mapping biopsy for preoperative identification of the longitudinal extension of BTC has been reported (11-13). On the other hand, the diagnostic accuracy of the POCS visual findings for indeterminate biliary lesions was higher than that of the POCS-guided forceps biopsy findings (14).

Although POCS plays important roles for diagnosis of indeterminate biliary strictures and for preoperative identification of the longitudinal extension of BTC, the diagnostic performance of POCS-guided forceps biopsy is not enough. The working channel of the cholangioscope is narrow, so only a mini-forceps with a $1.0-\mathrm{mm}$ diameter cup could be used for POCS-guided forceps biopsy. Therefore, only a small amount of specimen can be obtained by POCSguided biopsy. This is a possible reason for the inability to determine a pathological diagnosis and to evaluate the longitudinal tumor extent by POCS-guided forceps biopsy. In this study, we examined the adequate tissue acquisition rate of POCS-guided forceps biopsy for biliary lesions and that of POCS-guided forceps mapping biopsy for non-stenotic bile duct. We also evaluated the diagnostic performance of POCS-guided forceps biopsy for BTC and adverse events resulting from its use. We present the following article in accordance with the STROBE reporting checklist (available at http://dx.doi.org/10.21037/atm-20-2738).

\section{Methods}

\section{Study population}

Patients who underwent ERCP-related tissue acquisition with POCS for biliary disease between September 2016 and October 2018 at our hospital were retrospectively enrolled. Inclusion criteria were as follows: (I) patients who underwent POCS-guided forceps biopsy for indeterminate biliary lesion; (II) patients who underwent POCS-guided forceps mapping biopsy for BTC to identify longitudinal extension; (III) patients aged 20 years or older when the endoscopic procedures were performed. Exclusion criteria were as follows: (I) patients who did not provide consent; (II) patients who had surgically altered anatomy expect Billroth-I, and (III) patients who have been receiving chemotherapy for malignant diseases within one month prior to the acquisition of the pathological specimens.

Forty-seven patients with biliary disease were enrolled in the study. Participants included 32 men and 15 women aged 26-88 years (median age, 73 years). Twenty-eight patients had BTC and 19 had benign biliary lesions (Table 1). We defined Cohort 1 as patients who received POCS-guided forceps biopsy for biliary lesions. Cohort 2 was defined as the group of patients who received POCS-guided forceps biopsy for non-stenotic bile duct. Stenotic bile duct and non-stenotic bile duct was defined by fluoroscopy finding of ERCP. The biliary lesion sites with caliber changes in the biliary tract diameter were defined as stenotic bile ducts, and other sites were defined as non-stenotic bile duct. This study was performed according to the guidelines described in the World Medical Association Declaration of Helsinki statement (as revised in 2013) of ethical principles 
Table 1 Baseline characteristics of study patients

\begin{tabular}{|c|c|}
\hline Characteristics & $\begin{array}{l}\text { Biliary disease } \\
\qquad(\mathrm{n}=47)\end{array}$ \\
\hline Age [range], years & 73 [26-88] \\
\hline Sex, male/female & $32 / 15$ \\
\hline \multicolumn{2}{|l|}{ Location of stricture } \\
\hline Perihilar & 24 \\
\hline Distal & 19 \\
\hline Cystic duct & 2 \\
\hline Intrahepatic bile duct & 1 \\
\hline None & 1 \\
\hline Length of stricture, $\mathrm{mm}$ & $16.6(0-46.0)$ \\
\hline Acute cholangitis (presence/absence) & $12 / 35$ \\
\hline Total bilirubin, mg/dL & $1.3(0.3-14.8)$ \\
\hline \multicolumn{2}{|l|}{ Tumor marker } \\
\hline \multicolumn{2}{|l|}{ CEA, ng/mL } \\
\hline Malignant & $2.6(0.8-8.3)$ \\
\hline Benign & $2.2(0.9-10.1)$ \\
\hline \multicolumn{2}{|l|}{ CA19-9, U/mL } \\
\hline Malignant & $73.3(9.4-4,430.0)^{*}$ \\
\hline Benign & $9.3(0.8-309.5)$ \\
\hline Malignant & 28 \\
\hline Biliary tract cancer & 28 \\
\hline Benign & 19 \\
\hline Benign biliary stricture & 9 \\
\hline IgG4-associated sclerosing cholangitis & 3 \\
\hline Primary sclerosing cholangitis & 3 \\
\hline Drug-induced cholangitis & 2 \\
\hline Intraductal papillary neoplasm of bile duct & 1 \\
\hline Peribiliary cyst & 1 \\
\hline
\end{tabular}

Values are presented as number or median (range). *, $\mathrm{P}<0.001$ compared with the benign biliary disease using the MannWhitney U-test. CEA, carcinoembryonic antigen; CA19-9, carbohydrate antigen 19-9; IgG4, immunoglobulin G subclass 4 .

for medical research involving human subjects. The study protocol was also approved by the institutional review board of Tottori University (No. 18A178). In this retrospective study, informed consent was obtained from all participants using an opt-out approach.

\section{Endoscopic procedure}

A side-viewing duodenoscope (JF260V/TJF240V; Olympus Corp., Tokyo, Japan) and a 0.025 -inch hydrophilic guidewire (G-260-2545A; Olympus; MTA0025N48S; Medico's Hirata, Inc, Osaka, Japan; M00556700; Boston Scientific Corporation, Marlborough, MA, USA) were used during ERCP. POCS was performed using a mini endoscope (M00546600 SpyGlass DS Access; Boston Scientific) direct visualization system. We inserted a cholangioscope into the bile duct over the guidewire, and POCS-guided forceps biopsy under direct vision was performed with M00546270 (SpyBite Biopsy Forceps; Boston Scientific) with a 1.0-mm diameter cup (Figure 1).

Almost all patients underwent endoscopic sphincterotomy (EST) by using a sphincterotome (Olympus CleverCut KD-V411M-0725; Olympus), if it was not previously performed. Eighteen patients in the study group had previously undergone EST. Endoscopic papillary balloon dilation (EPBD) was performed for 4 cases using a balloon dilatation catheter (ZR25-08-23, RN25-0630-18; KANEKA Medix Corporation, Osaka, Japan) because they received anti-thrombotic therapy. One patient underwent a precut papillotomy by using a needle knife (9913023121; MTW-Endoskopie W. Haag KG, Wesel, Germany).

\section{Diagnostic criteria and definition of adequate tissue}

The diagnosis of BTC was based on the pathological diagnosis of bile aspiration cytology, and transpapillary forceps biopsy, endoscopic ultrasonography-guided fine needle aspiration (EUS-FNA), or surgical specimen. Patients with benign disease had a final diagnosis based on clinical and radiological follow-up data. Biopsy specimens were stained with hematoxylin and eosin and, if necessary, immunostaining, including $\mathrm{p} 53$ and $\mathrm{Ki}-67$, was also performed. Adequate tissue was defined as a specimen that allowed the evaluation of the histological finding of the biliary epithelium. A specimen that contained only interstitial tissue was deemed insufficient (Figure 2). Malignancy or suspected malignancy was considered positive in histological findings.

We evaluated the adequate tissue acquisition rate of POCS-guided forceps biopsy for biliary strictures in Cohort 1 and that of POCS-guided forceps mapping biopsy for 

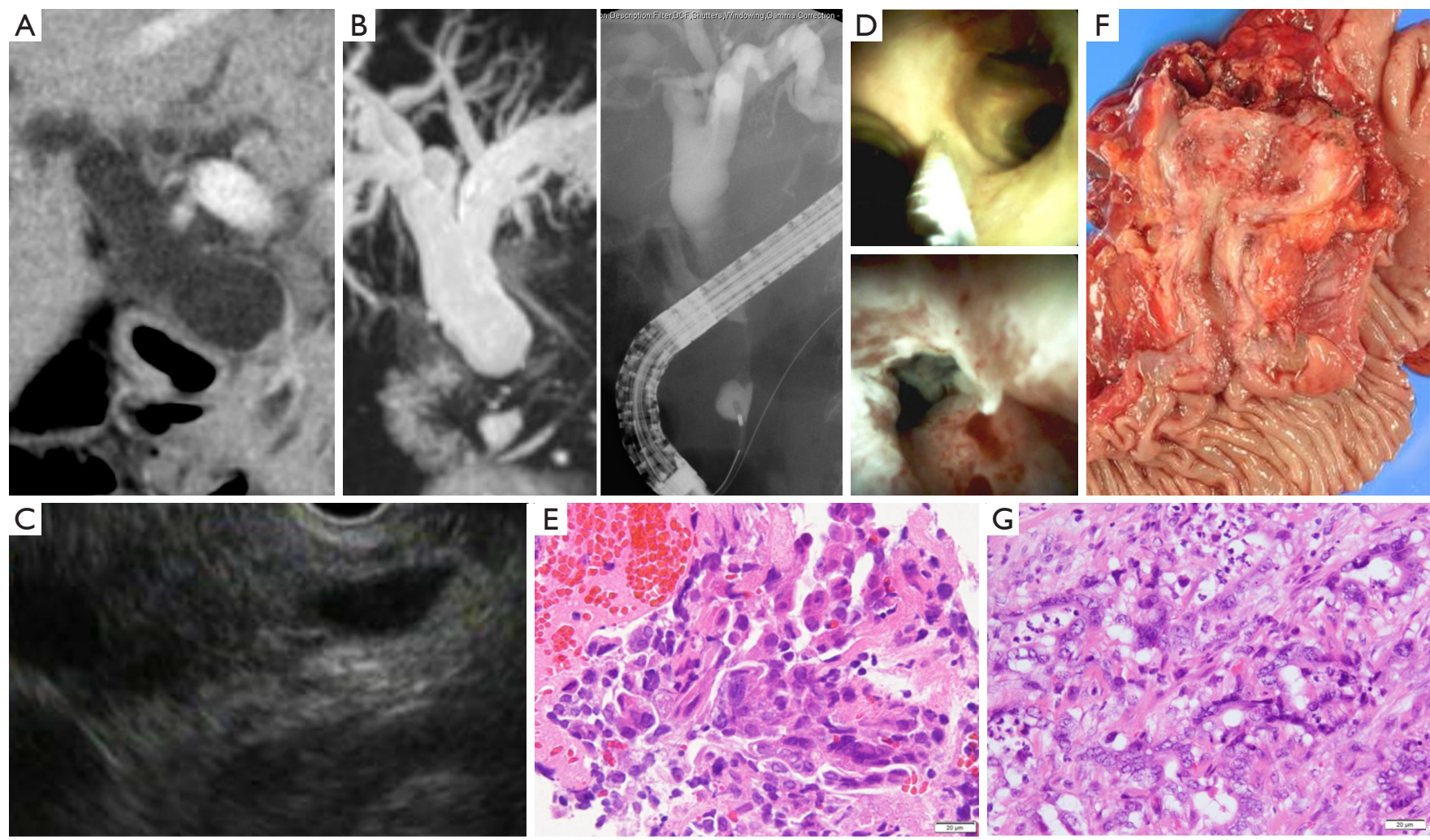

Figure 1 A case of bile duct stricture diagnosed as distal cholangiocarcinoma with peroral cholangioscopy (POCS)-guided forceps biopsy. (A) Computed tomography scan revealed an irregular nodule in the distal bile duct; (B) magnetic resonance cholangiopancreatography and endoscopic retrograde cholangiography showed stenosis in the distal bile duct; (C) endoscopic ultrasonography showed an irregular nodule in the distal bile duct; (D) POCS revealed the irregular granular mucosa that existed distal bile duct. POCS-guided forceps biopsy was performed for the biliary stricture in the distal bile duct and for the non-stenotic extrahepatic and intrahepatic bile ducts to identify the longitudinal extension margin of the cholangiocarcinoma; (E) hematoxylin and eosin staining revealed adenocarcinoma in specimens obtained from the biliary stricture; (F) this patient underwent pancreatoduodenectomy; and (G) this patients was diagnosed with distal cholangiocarcinoma histologically (hematoxylin and eosin stain). POCS, peroral cholangiography.

non-stenotic bile duct in Cohort 2. The factors affecting adequate tissue acquisition of POCS-guided forceps biopsy in each cohort were also evaluated. We evaluated the pathological diagnostic performance of the POCS-guided forceps biopsy for BTC. We also evaluated adverse events of POCS-guided forceps biopsy for biliary disease.

\section{Statistical analysis}

Statistical analysis was performed using StatFlex ver. 7.0 for Windows (Artec Corp., Osaka, Japan). Categorical variables were compared using the chi-squared test, and continuous variables were compared using the MannWhitney U-test. All values are expressed as mean \pm standard deviation or median with interquartile range. Subgroup analyses of age ( $<65$ or $\geq 65$ years), sex, malignant biliary disease, the location of the biliary lesion or stricture (distal or non-distal), length of biliary stricture ( $<15$ or $\geq 15 \mathrm{~mm}$ ), macroscopic types of BTC (flat type or non-flat type composed of nodular and papillary type), presence of acute cholangitis, level of serum total bilirubin (T-Bil, $<1.5$ or $\geq 1.5 \mathrm{mg} / \mathrm{dL}$ ), level of carcinoembryonic antigen (CEA, $<5.0$ or $\geq 5.0 \mathrm{ng} / \mathrm{mL}$ ), level of carbohydrate antigen $19-9$ (CA19-9, $<37$ or $\geq 37 \mathrm{U} / \mathrm{mL}$ ), procedure time ( $\leq 75$ or $>75$ minutes), EST, and previous biliary stenting before POCS-guided forceps biopsy were assessed to determine the adequate tissue acquisition rate of POCS-guided forceps biopsy. In Cohort 2, biopsy site (bifurcation of the bile duct or not, intrahepatic bile duct or extrahepatic bile duct, right side or left side) was also included in the subgroup analysis. 

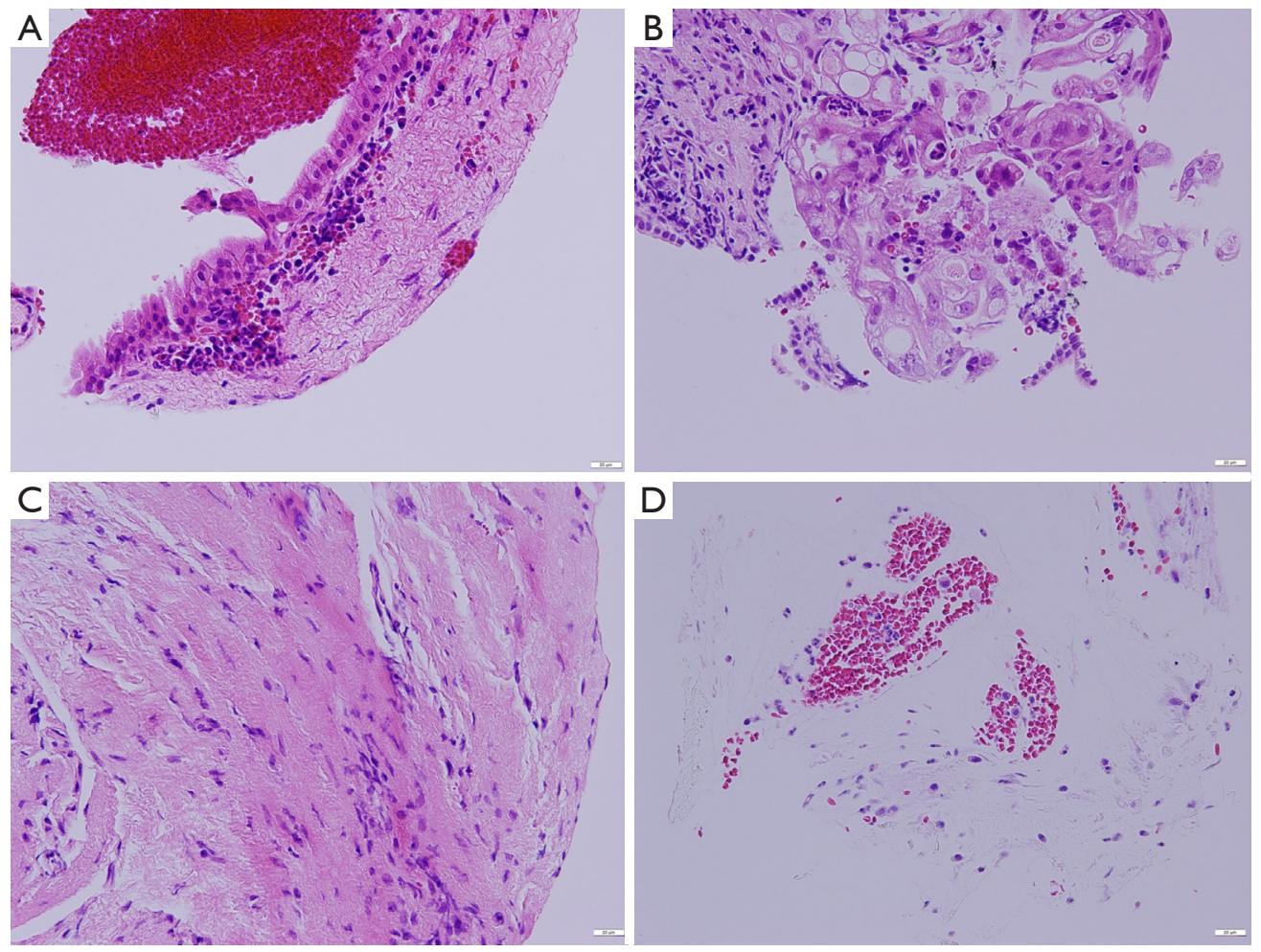

Figure 2 Histopathological findings of specimens obtained by peroral cholangioscopy-guided forceps biopsy. (A) An adequate specimen containing biliary epithelium without atypia is shown (normal). (B) An adequate specimen with biliary epithelial cells with severe atypia is revealed (adenocarcinoma). (C) An insufficient specimen containing interstitial tissue is shown. (D) An insufficient specimen containing only hepatocytes is revealed. All of the specimens were stained with hematoxylin and eosin.

We carried out univariate analyses to assess the adequate tissue acquisition rate, and factors with $\mathrm{P}<0.1$ were included in the multivariate logistic regression analyses. The significance level was set at $\mathrm{P}<0.05$. In multivariate logistic regression analyses, incomplete cases with the missing values of candidate factors were excluded.

\section{Results}

\section{Patient's characteristics and baseline evaluation}

Table 1 showed the characteristics of patients with biliary disease. In this study, the malignant group included 12 patients with perihilar cholangiocarcinoma, 14 patients with distal cholangiocarcinoma, one patient with intrahepatic cholangiocarcinoma and one patient with cystic ductal carcinoma. Macroscopic types of BTC included four papillary-type, 16 nodular-type, and eight flat-type. The benign group included nine patients with benign biliary strictures, three patients with IgG4-associated sclerosing cholangitis, three patients with primary sclerosing cholangitis, two patients with drug-induced cholangitis, one with intraductal papillary neoplasm of the bile duct (IPNB), and one with a peribiliary cyst. This study group included 24 patients with perihilar bile duct stricture, 19 patients with distal bile duct stricture, two patients with cystic duct stricture, and one patient with intrahepatic bile duct stricture. One patient did not have any biliary stricture, and he received POCS-guided forceps biopsy for distal bile duct near the ampulla of Vater in order to evaluate the hypertrophic extrahepatic bile duct. The median length of stricture in this study group was $16.6 \mathrm{~mm}$ (range, 0-46.0 mm). The median levels of serum T-Bil, CEA, and CA19-9 were $1.3 \mathrm{mg} / \mathrm{dL}$ (range, $0.3-14.8 \mathrm{mg} / \mathrm{dL}$ ), $2.4 \mathrm{ng} / \mathrm{mL}$ (range, $0.8-10.1 \mathrm{ng} / \mathrm{mL}$ ), and $34.6 \mathrm{U} / \mathrm{mL}$ (range, $0.8-4,430.0 \mathrm{U} / \mathrm{mL}$ ), respectively (Table 1). The median procedure time of POCS-guided forceps biopsy was 77 minutes (range, 26-170 minutes). The final clinical diagnosis was derived from surgical pathology in 15 patients. 


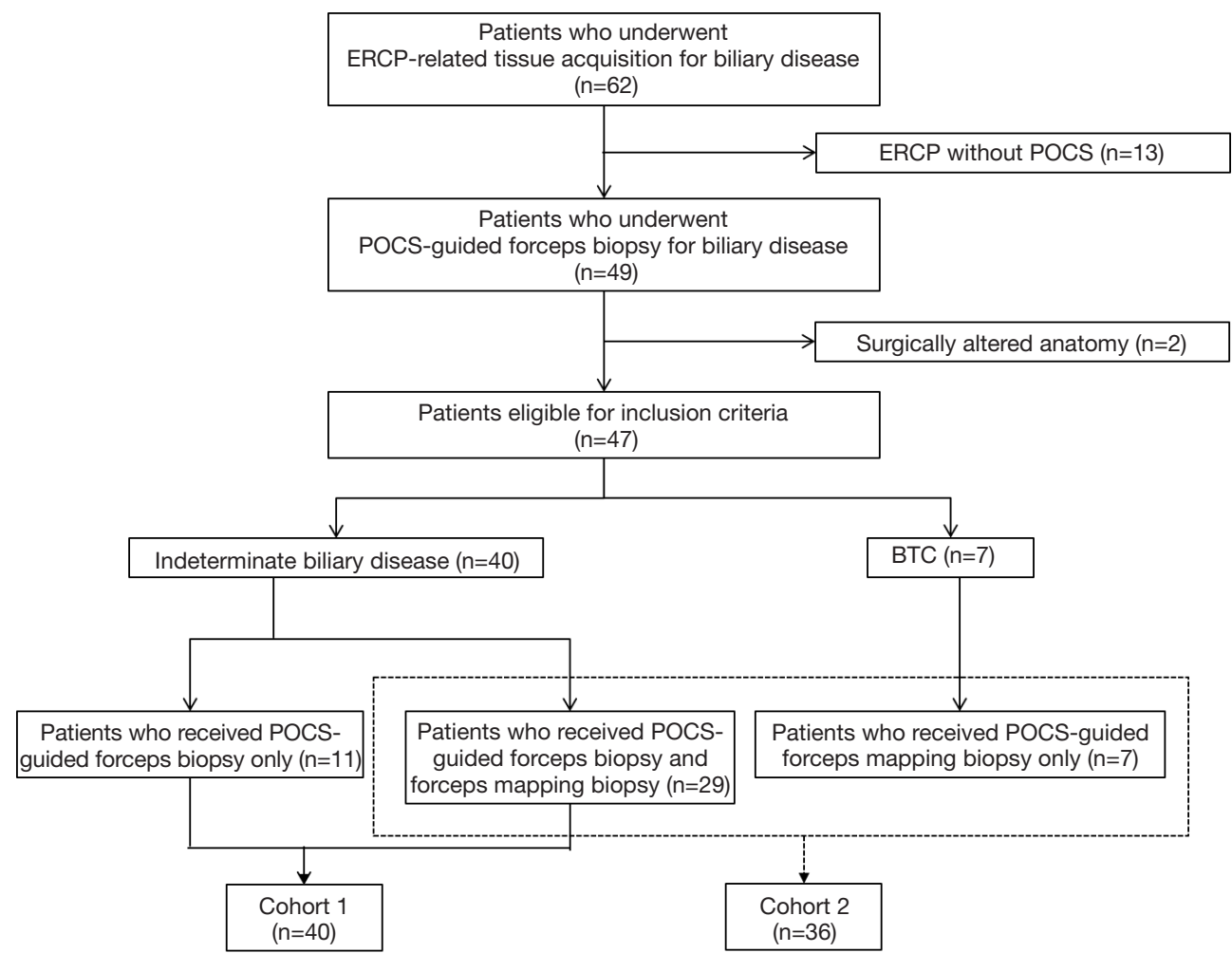

Figure 3 Flowchart of included patients in the study. ERCP, endoscopic retrograde cholangiopancreatography; POCS, peroral cholangioscopy; BTC, biliary tract cancer.

There were 18 patients with a final diagnosis based on clinical and radiological follow-up data (median follow up period, 7 months; range, 1-22 months). We performed both the POCS-guided forceps biopsy for biliary lesions and the POCS-guided forceps mapping biopsy for 29 patients with biliary disease. Eleven patients received only the POCSguided forceps biopsy for indeterminate biliary lesions, and Seven patients received only the POCS-guided forceps mapping biopsy for non-stenotic bile duct to determine the longitudinal extension of BTC. Therefore, Cohort 1 and Cohort 2 included 40 and 36 patients, respectively (Figure 3).

\section{Diagnostic performance and tissue acquisition rate of POCS-guided forceps biopsy for indeterminate biliary lesion}

The total and the median number of biopsies for indeterminate biliary lesions were 140 and 3 (range, 2-8) in Cohort 1, respectively. The total number of POCSguided forceps mapping biopsies was 196 in Cohort 2. By the location in the bile duct, 93 specimens were obtained from the perihilar bile ducts of 25 patients (41 specimens from the confluence of the hepatic ducts of 23 patients), 31 specimens from the distal bile ducts of 15 patients, 22 specimens from the junction of the cystic ducts of 11 patients, 27 specimens from the first order branch of the intrahepatic ducts of 12 patients, and 23 specimens from the second order branch of the intrahepatic ducts of 12 patients. Eleven patients received biliary stenting before POCS-guided forceps biopsy. In Cohort 1, 21 patients were with BTC, and 19 patients were with benign. Cohort 1 included 22 patients with perihilar bile duct stricture, 15 patients with distal bile duct stricture, 2 patients with cystic duct stricture, and 1 patient with extrahepatic bile duct hypertrophy. Figure 4 showed diagnostic flowchart of indeterminate biliary disease and Table 2 summarizes the diagnostic performance of POCS-guided forceps biopsy to differentiate BTC from benign biliary disease. The values for sensitivity, specificity, positive predictive value (PPV), negative predictive value (NPV), and the accuracy of 


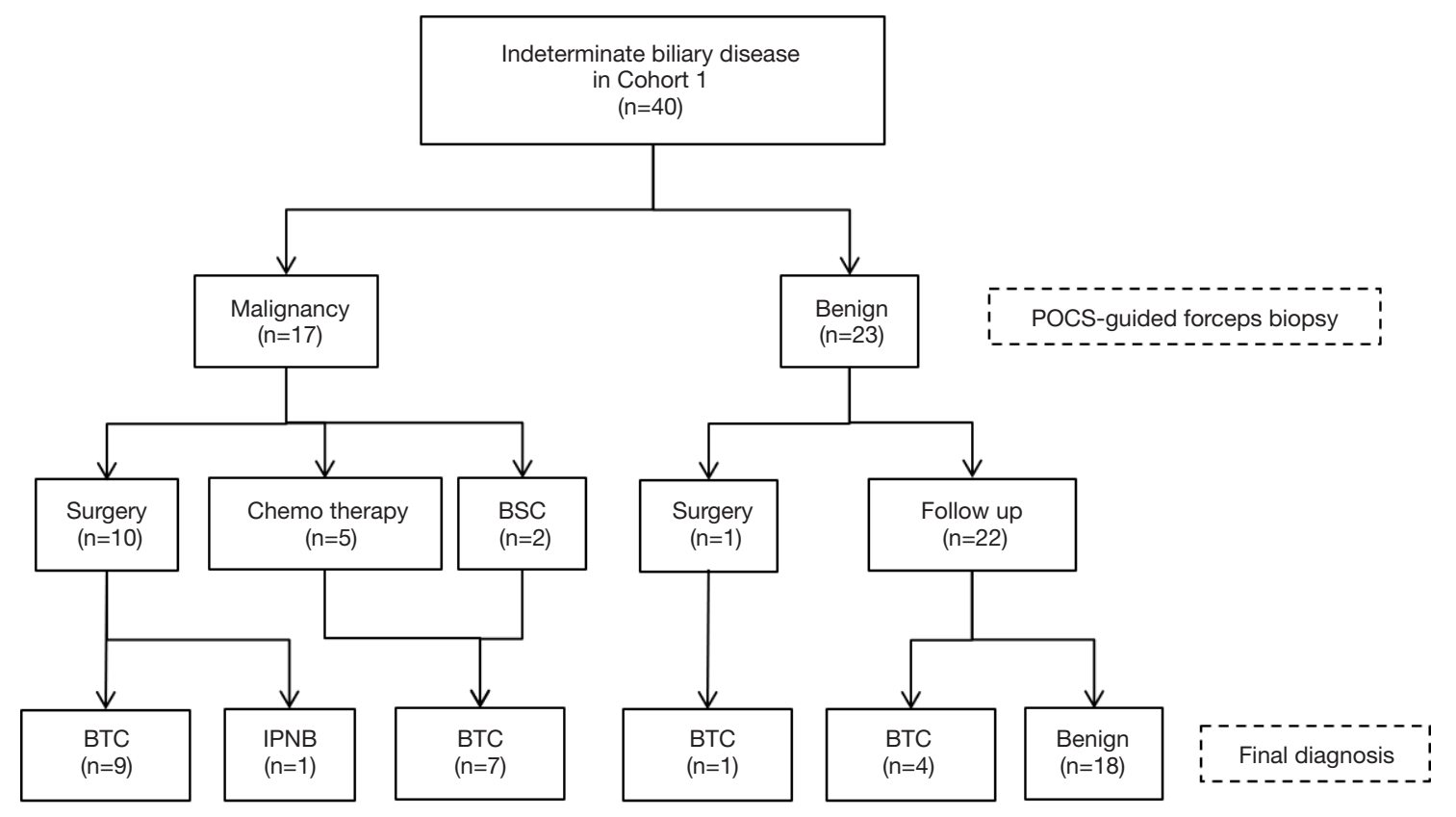

Figure 4 Diagnostic flowchart of patients with indeterminate biliary disease in the study. BTC, biliary tract cancer; POCS, peroral cholangioscopy; BSC, best supportive care; IPNB, intraductal papillary neoplasm of the bile duct.

Table 2 Diagnostic ability of peroral cholangioscopy-guided forceps biopsy for differentiating biliary tract cancer from benign biliary disease

\begin{tabular}{lccccc}
\hline & Sensitivity, $\%$ & Specificity, $\%$ & PPV, \% & NPV, \% & Accuracy, \% \\
\hline POCS-guided forceps & $76.2(16 / 21)$ & $94.7(18 / 19)$ & $94.1(16 / 17)$ & $78.3(18 / 23)$ & $85.0(34 / 40)$ \\
\hline
\end{tabular}

POCS, peroral cholangioscopy; PPV, positive predictive value; NPV, negative predictive value.

POCS-guided forceps biopsy were 76.2\%, 94.7\%, 94.1\%, $78.3 \%$, and $85.0 \%$, respectively (Table 2). The adequate tissue acquisition rate of POCS-guided forceps biopsy for biliary lesions was $86.4 \%(121 / 140)$.

\section{Factors affecting the adequate tissue acquisition of POCS- guided forceps biopsy for indeterminate biliary lesion}

Table 3 summarizes the result of analyses for the adequate tissue acquisition in POCS-guided forceps biopsy for biliary lesions. In the univariate analyses, age, sex, the location of the biliary lesion, and previous biliary stenting before POCS were factors with $\mathrm{P}<0.1$ and were included in the multivariate logistic regression analyses. Age $<65$ years old [odds ratio $0.170,95 \%$ confidence interval (CI), 0.044 $0.649, \mathrm{P}=0.010$ ] and previous biliary stenting before POCS (odds ratio $0.199,95 \% \mathrm{CI}, 0.053-0.756, \mathrm{P}=0.017$ ) were the significant factors affecting the adequate tissue acquisition rate in the multivariate analysis.

\section{Tissue acquisition rate of POCS-guided forceps biopsy for non-stenotic bile duct}

Cohort 2 included 17 patients with perihilar bile duct stricture, 16 patients with distal bile duct stricture, two patients with cystic duct stricture, and one patient with intrahepatic bile duct lesion. The adequate tissue acquisition rate of POCS-guided forceps mapping biopsy for non-stenotic bile duct was $68.9 \%(135 / 196)$, which was significantly lower than that of POCS-guided forceps biopsy for biliary lesion $(\mathrm{P}<0.001)$. The adequate tissue acquisition rates of POCS-guided forceps mapping biopsy were calculated by the location in the bile duct: the distal bile duct (58.1\%), the junction of the cystic duct (86.4\%), the perihilar bile duct $(77.4 \%$; including the confluence of the hepatic duct $82.9 \%$ ), the first order branch of the 
Table 3 Factors affecting the adequate tissue acquisition of peroral cholangioscopy-guided forceps biopsy for biliary lesions

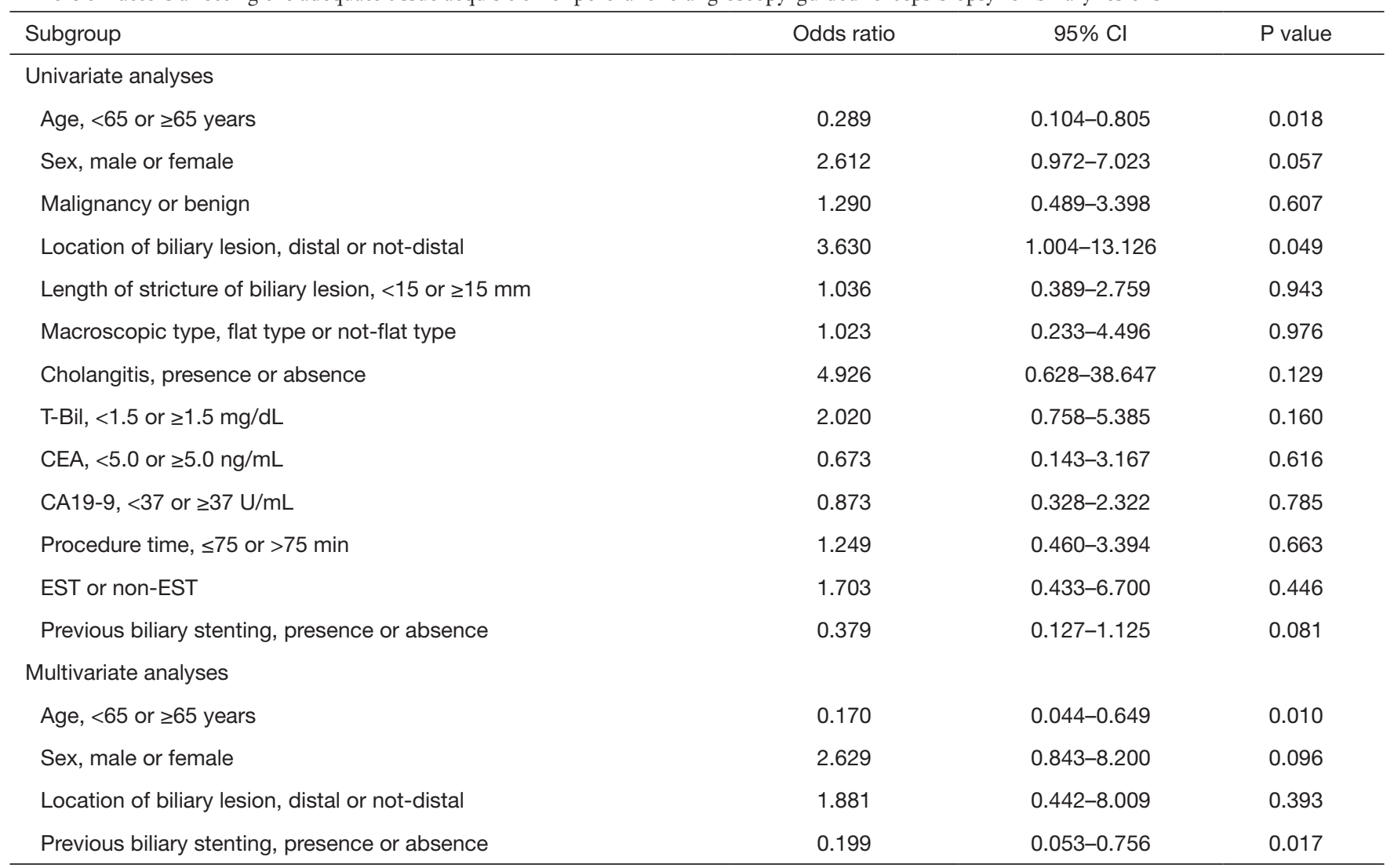

$\mathrm{P}$ value: logistic regression model. $\mathrm{Cl}$, confidence interval; T-Bil, total bilirubin; CEA, carcinoembryonic antigen; CA19-9, carbohydrate antigen; EST, endoscopic sphincterotomy.

intrahepatic bile duct (37.0\%), and the second order branch of the intrahepatic bile duct (69.6\%) (Table 4).

\section{Diagnostic ability of POCS-guided forceps mapping biopsy for evaluation of the lateral extension of BTC}

Thirteen patients received surgical resection for BTC in cohort 2 (Figure 5). Nine patients underwent pancreatoduodenectomy, 2 underwent hepatectomy with extrahepatic bile duct resection, and 2 received extrahepatic bile duct resection with cholecystectomy. Three patients whose surgical margins were positive at the hepatic side and 2 of them could not be evaluated the diagnostic accuracy of POCS-guided forceps mapping biopsy for the lateral extension of BTC. Eight patients did not undergo POCSguided forceps mapping biopsy to evaluate the lateral extension at duodenal side. The specimens obtained from the hepatic side of BTC were insufficient in 2 patients, and those from the duodenal side were also insufficient in 2 patients. The diagnostic accuracy of lateral extent at hepatic side, and duodenal side were $63.6 \%$ (7/11), and $40.0 \%(2 / 5)$, respectively.

\section{Factors affecting the adequate tissue acquisition of POCS- guided forceps biopsy for non-stenotic bile duct}

Table 5 summarizes the results of the analyses for the adequate tissue acquisition in POCS-guided forceps mapping biopsy for a non-stenotic bile duct. In the univariate analyses, age, malignant lesion, the location of the biliary lesion, the length of stricture, the biopsy site in the intrahepatic bile duct, procedure time, and EST were factors with $\mathrm{P}<0.1$, and were included in the multivariate logistic regression analyses. The biliary lesion in the distal bile duct (odds ratio 0.322, 95\% CI, 0.139-0.744, $\mathrm{P}=0.008$ ), procedure time of $75 \mathrm{~min}$ or less (odds ratio 3.012, $95 \%$ 
Table 4 The adequate tissue acquisition rate of peroral cholangioscopy-guided forceps biopsy for biliary lesions and nonstenotic bile duct

\begin{tabular}{|c|c|}
\hline Biopsy site & $\begin{array}{l}\text { Adequate tissue } \\
\text { acquisition rate, \% }\end{array}$ \\
\hline Biliary lesions & $86.4(121 / 140)$ \\
\hline Non-stenotic bile duct & $68.9(135 / 196)$ \\
\hline Distal bile duct & $58.1(18 / 31)$ \\
\hline Junction of the cystic duct & $86.4(19 / 22)$ \\
\hline Perihilar bile duct & $77.4(72 / 93)$ \\
\hline Confluence of the hepatic duct & $82.9(34 / 41)$ \\
\hline Right hepatic duct & $75.0(12 / 16)$ \\
\hline Left hepatic duct & $70.4(19 / 27)$ \\
\hline Branch of intrahepatic bile duct & $52.0(26 / 50)$ \\
\hline First order branch of intrahepatic bile duct & $37.0(10 / 27)$ \\
\hline $\begin{array}{l}\text { Right first order branch of intrahepatic } \\
\text { bile duct }\end{array}$ & $29.4(5 / 17)$ \\
\hline $\begin{array}{l}\text { Left first order branch of intrahepatic bile } \\
\text { duct }\end{array}$ & $50.0(5 / 10)$ \\
\hline $\begin{array}{l}\text { Second order branch of intrahepatic bile } \\
\text { duct }\end{array}$ & $69.6(16 / 23)$ \\
\hline $\begin{array}{l}\text { Right second order branch of intrahepatic } \\
\text { bile duct }\end{array}$ & $66.7(4 / 6)$ \\
\hline $\begin{array}{l}\text { Left second order branch of intrahepatic } \\
\text { bile duct }\end{array}$ & $70.6(12 / 17)$ \\
\hline
\end{tabular}

POCS, peroral cholangioscopy.

CI, 1.092-8.312, $\mathrm{P}=0.033$ ), and EST (odds ratio 7.041, 95\% CI, 2.117-23.421, P=0.002) were the significant factors affecting the adequate tissue acquisition rate in the multivariate analyses.

\section{Adverse events}

Adverse events following POCS-guided forceps biopsy occurred in eight patients $(17.0 \%)$, with four patients $(8.5 \%)$ developing acute pancreatitis, including one case of severe pancreatitis; and three patients $(6.4 \%)$ developing cholangitis. Severe hemorrhage related to the precut papillotomy occurred in one patient $(2.1 \%)$. No perforations were observed, all cases were resolved with conservative treatment, and there was no procedure-related mortality (Table 6).

\section{Discussion}

In recent years, POCS has become a widely-used method for the diagnosis of indeterminate biliary stricture. POCS allows optical viewing of the biliary system, as well as targeted forceps biopsies under direct vision. Some studies reported that the use of POCS can improve the diagnostic accuracy of indeterminate biliary lesions $(6,12)$. On the other hands, the sensitivities of POCS-guided forceps biopsy for malignant biliary stricture $(60.1 \%)$ and cholangiocarcinoma $(66.2 \%)$ were still insufficient (8) and were similar for fluoroscopic tissue acquisition $(7,15)$. Moreover, the sensitivity and accuracy of POCSguided forceps biopsy for indeterminate biliary lesions were lower than those of visual findings for POCS in past study (13). Because of the narrow working channel of the cholangioscope, only a mini-forceps with a $1.0-\mathrm{mm}$ diameter cup was used for the diagnosis in POCS-guided forceps biopsy so that the specimen obtained by POCS is relatively small. A small amount of tissue sampling by POCSguided forceps biopsy may be related to the insufficient pathological diagnostic ability of that for indeterminate biliary lesions. In the tissue amount, less tissue was obtained from POCS-guided forceps biopsy than fluoroscopyguided biopsy (16). Indeed, POCS-guided tissue acquisition may only result in an insufficient amount of tissue. In our study, the adequate tissue acquisition rate of POCS-guided forceps biopsy for biliary lesions was acceptable (86.4\%), and there were only 3 patients with biliary lesions in which an adequate amount of tissue could not be obtained. Meanwhile, the sensitivity of POCS-guided forceps biopsy for BTC was not acceptable (76.2\%), and it was unsatisfactory compared to the adequate tissue acquisition rate. A working channel with a larger diameter for larger capacity forceps might improve the diagnostic utility for the indeterminate biliary lesion (17). In the multivariate logistic regression analyses, we found that patients under 65 years old and the previous biliary stenting before POCS were factors affecting low tissue acquisition rate. We could not understand why the low tissue acquisition rate of POCSguided forceps biopsy was in patients under 65 years old. Meanwhile, the biliary epithelium might be detached from the biliary tract by the previous biliary stenting, so that we could not sample the specimens including epithelium of biliary tract. In any case with POCS-guided forceps biopsy for biliary lesion, it may be better to avoid previous biliary stenting as much as possible except for patients with acute 


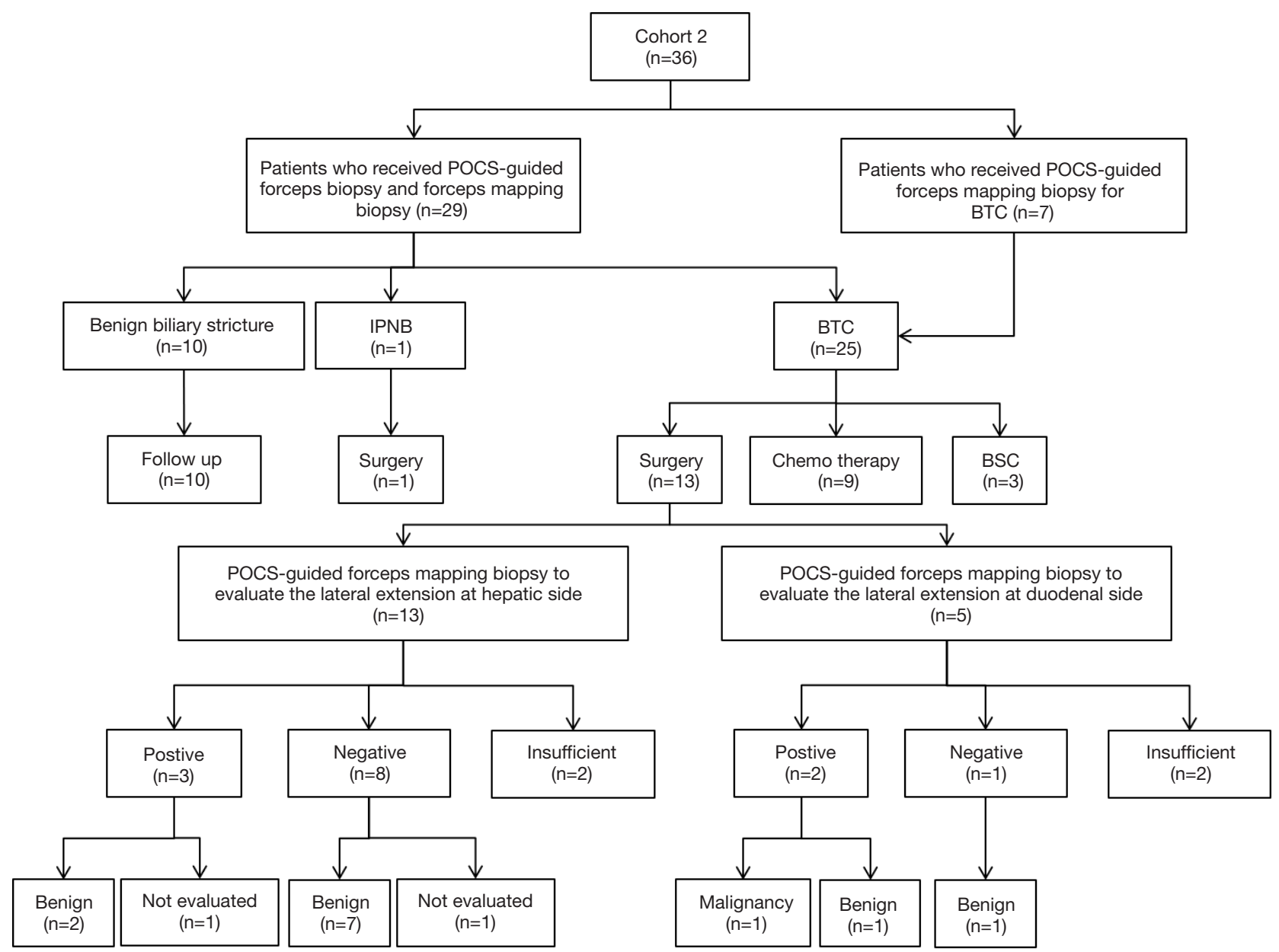

Figure 5 Flowchart of patients who underwent peroral cholangioscopy-guided forceps mapping biopsy in the study. BTC, biliary tract cancer; POCS, peroral cholangioscopy; BSC, best supportive care; IPNB, intraductal papillary neoplasm of the bile duct.

obstructive suppurative cholangitis. If it was impossible, multiple biopsies or combination of other tissue acquisition methods, such as biliary brush cytology, and forceps biopsy in fluoroscopy, might be needed. In our study, we also performed multiple biopsies (median number of biopsies, 3; range, 2-8) for all cases with indeterminate biliary lesions. Although we did not evaluate the factors affecting the diagnostic accuracy of POCS-guided forceps biopsy for BTC, 4 times or more biopsies might be needed to increase the diagnostic accuracy of POCS-guided forceps biopsy for BTC. Moreover, to compare the specimen obtained by POCS-guided forceps biopsy, that of the other methods are larger in general (16). Navaneenthan et al. reported that a combination of biliary brush cytology and forceps biopsy modestly increased the sensitivity to $59.4 \%$ (5). Some combinations of other tissue acquisition methods should be performed as much as possible if the cost and the additional endoscopic procedure time are acceptable.

Superficial intraductal spread, which is a feature of BTC, is present in $14.6 \%$ of patients with BTC in previous studies $(3,10)$. The presence of superficial intraductal spread is related to positive resection margins, local recurrence, and poor prognosis after surgery. Therefore, it is essential to identify the exact perihilar and distal margins of the preoperative BTC. Hijioka et al. reported the usefulness of fluoroscopic mapping biopsy procedures to distinguish between benign and malignant foci (18). However, that method did not allow targeted forceps biopsies under direct vision of the biliary system, and it was uncertain whether the biopsy site was true or not. Meanwhile, POCS-guided forceps mapping biopsy allowed targeted forceps biopsy under direct and fluoroscopic vision of the biliary system. 
Table 5 Factors affecting the adequate tissue acquisition of peroral cholangioscopy-guided forceps biopsy for non-stenotic bile duct

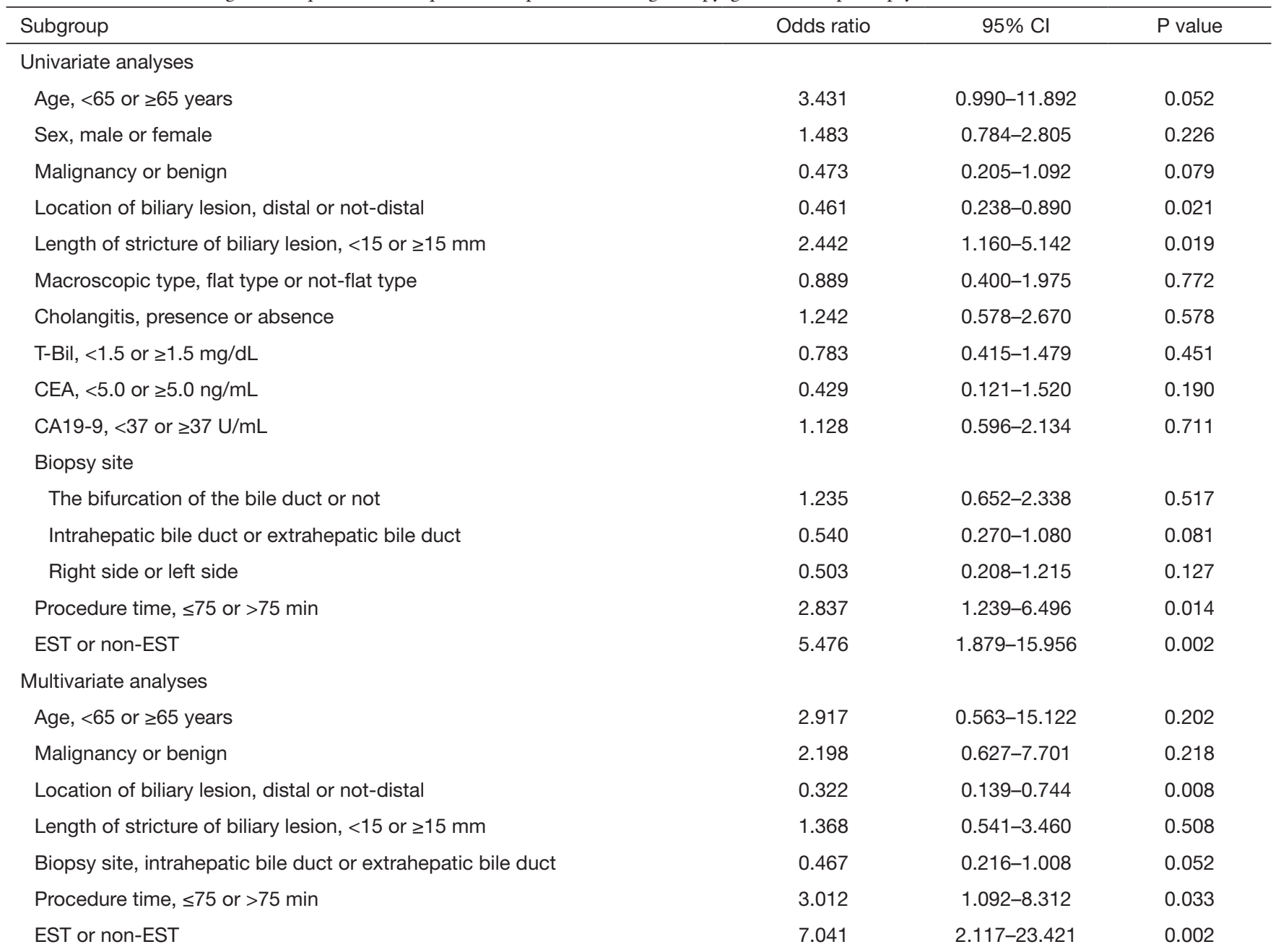

$P$ value: logistic regression model. $\mathrm{Cl}$, confidence interval; T-Bil, total bilirubin; CEA, carcinoembryonic antigen; CA19-9, carbohydrate antigen; EST, endoscopic sphincterotomy.

Table 6 The adverse event of peroral cholangioscopy-guided forceps biopsy

\begin{tabular}{lc}
\hline Adverse event & POCS-guided forceps biopsy $(\mathrm{n}=47)$ \\
\hline Pancreatitis & $8.5 \%(4 / 47)$ \\
Bleeding & $2.1 \%(1 / 47)$ \\
Infection & $6.4 \%(3 / 47)$ \\
Perforation & 0 \\
Cardiac & 0 \\
Pulmonary & 0 \\
Medication reaction & 0 \\
Other & 0 \\
Overall & $17.0 \%(8 / 47)$ \\
\hline
\end{tabular}

POCS, peroral cholangioscopy.
Although recent studies reported the usefulness of POCSguided forceps biopsy in the preoperative assessment of the longitudinal extension of BTC, the sensitivity of that was not necessarily sufficient $(12,13,19)$. In our study, the adequate tissue acquisition rate of POCS-guided forceps biopsy for non-stenotic bile duct was also insufficient (68.9\%). In addition, the diagnostic accuracy of POCSguided forceps mapping biopsy for evaluation of lateral extent of BTC was also unsatisfactory. In particular, 3 of 5 cases diagnosed as positive by POCS-guided forceps mapping biopsy were benign eventually. In clinical practice, the careful and accurate procedure of POCS-guided forceps mapping biopsy is required to obtain the specimens from desired sites of biliary tracts. Mapping biopsies 
could not be performed from the desired sites so that the contamination of specimens from other sites might occur at times. Moreover, the contamination of the detached biliary epithelium from BTC might also occur. Reasons for the insufficient tissue acquisition rate and diagnostic accuracy of POCS-guided forceps mapping biopsy may be not only the small amount of specimen obtained by POCSguided forceps biopsy but also the technical difficulty of the limited flexion of the cholangioscope tip, especially in lesions above the biliary stricture as it may be inadequate to target the desired biopsy site (20). Furthermore, the desired biopsy site above the curvature of the biliary tract might be a negative factor in the adequate tissue acquisition. Therefore, we think it is important to evaluate the factors affecting the adequate tissue acquisition of POCS-guided forceps biopsy.

In previous studies, there were a few reports about the factors affecting the accuracy or sensitivity of ERCPrelated tissue acquisition for BTC or malignant biliary stricture (21-23). However, no study reported on the factors affecting the adequate tissue acquisition of ERCPrelated tissue acquisition, including POCS-guided forceps biopsy, for BTC or malignant biliary stricture. Therefore, we selected some candidate factors related to the adequate tissue acquisition from the factors affecting the accuracy or sensitivity of ERCP-related tissue acquisition for BTC or malignant biliary stricture.

In multivariate logistic regression analyses, we found that the presence of the lesion in the distal bile duct and the POCS-guided forceps mapping biopsy without EST were factors affecting a low tissue acquisition rate. The reason for those might be the limited flexion of the cholangioscope tip making it difficult to target the desired biopsy site. Multiple biopsies might be needed to improve the adequate tissue acquisition of POCS-guided forceps mapping biopsy for patients with distal bile duct lesions. It is uncertain why the procedure time of 75 minutes or less was a factor affecting a high tissue acquisition rate of POCS-guided forceps mapping biopsy. Speculation includes that the biliary epithelium may be detached from the biliary tract as the procedure time of POCS increases and that the specimen which can be evaluated for a histological finding of the biliary epithelium may be lost. In any case, it is difficult to shorten the procedure time of POCS-guided forceps biopsy and increase the sampling. A more flexible cholangioscope with a wide working channel and a larger capacity forceps might help to improve the adequate tissue acquisition rate.

This study has some limitations. First, this was a retrospective analysis of a small number of cases in single-center. Second, patients who were diagnosed through clinical follow-up were also included in this study. Therefore, it was unclear whether the cases with negative pathological findings were truly benign biliary disease or not. A prospective long-term study including a larger number of patients is required to strengthen the investigation.

In conclusions, previous biliary stenting was a factor affecting a low tissue acquisition rate of POCS-guided forceps biopsy for the biliary lesion. In the POCS-guided forceps mapping biopsy for non-stenotic bile duct, the presence of the lesion in the distal bile duct, the POCSguided forceps mapping biopsy without EST, and the long procedure time of POCS were factors affecting low tissue acquisition rates.

\section{Acknowledgments}

We wish to thank our colleagues in the Departments of Gastroenterology and Hepatology, and Pathology at Tottori University Faculty of Medicine (Tottori, Japan).

Funding: None.

\section{Footnote}

Reporting Checklist: The authors have completed the STROBE reporting checklist. Available at http://dx.doi. org/10.21037/atm-20-2738

Data Sharing Statement: Available at http://dx.doi. org/10.21037/atm-20-2738

Conflicts of Interest: All authors have completed the ICMJE uniform disclosure form (available at http://dx.doi. org/10.21037/atm-20-2738). The authors have no conflicts of interest to declare.

Ethical Statement: The authors are accountable for all aspects of the work in ensuring that questions related to the accuracy or integrity of any part of the work are appropriately investigated and resolved. The study was conducted in accordance with the Declaration of Helsinki (as revised in 2013). The clinical research was approved by the institutional review board of Tottori University (No. 18A178). In this retrospective study, informed consent was obtained from all participants using an optout approach. 
Open Access Statement: This is an Open Access article distributed in accordance with the Creative Commons Attribution-NonCommercial-NoDerivs 4.0 International License (CC BY-NC-ND 4.0), which permits the noncommercial replication and distribution of the article with the strict proviso that no changes or edits are made and the original work is properly cited (including links to both the formal publication through the relevant DOI and the license). See: https://creativecommons.org/licenses/by-nc-nd/4.0/.

\section{References}

1. Flemming JA, Zhang-Salomons J, Nanji S, et al. Increased incidence but improved median overall survival for biliary tract cancers diagnosed in Ontario from 1994 through 2012: A population-based study. Cancer 2016;122:2534-43.

2. Ishihara S, Horiguchi A, Miyakawa S, et al. Biliary tract cancer registry in Japan from 2008 to 2013. J Hepatobiliary Pancreat Sci 2016;23:149-57.

3. Wakai T, Shirai Y, Sakata J, et al. Clinicopathological features of benign biliary strictures masquerading as biliary malignancy. Am Surg 2012;78:1388-91.

4. Burnett AS, Calvert TJ, Chokshi RJ. Sensitivity of endoscopic retrograde cholangiopancreatography standard cytology: 10-y review of the literature. J Surg Res 2013;184:304-11.

5. Navaneethan U, Njei B, Lourdusamy V, et al. Comparative effectiveness of biliary brush cytology and intraductal biopsy for detection of malignant biliary strictures: A systematic review and meta-analysis. Gastrointest Endosc 2015;81:168-76.

6. Draganov PV, Chauhan S, Wagh MS, et al. Diagnostic accuracy of conventional and cholangioscopy-guided sampling of indeterminate biliary lesions at the time of ERCP:A prospective, long-term follow-up study. Gastrointest Endosc 2012;75:347-53.

7. Walter D, Peveling-Oberhag J, Schulze F, et al. Intraductal biopsies in indeterminate biliary stricture: Evaluation of histopathological criteria in fluoroscopyvs. cholangioscopy guided technique. Dig Liver Dis 2016;48:765-70.

8. Navaneethan U, Hasan MK, Lourdusamy V, et al. Singleoperator cholangioscopy and targeted biopsies in the diagnosis of indeterminate biliary strictures: A systematic review. Gastrointest Endosc 2015;82:608-14.e2.

9. Wakai T, Shirai Y, Moroda T, et al. Impact of ductal resection margin status on long-term survival in patients undergoing resection for extrahepatic cholangiocarcinoma.
Cancer 2005;103:1210-6.

10. Igami T, Nagino M, Oda K, et al. Clinicopathologic study of cholangiocarcinoma with superficial spread. Ann Surg 2009;249:296-302.

11. Lee SS, Kim MH, Lee SK, et al. MR cholangiography versus cholangioscopy for evaluation of longitudinal extension of hilar cholangiocarcinoma. Gastrointest Endosc 2002;56:25-32.

12. Osanai M, Itoi T, Igarashi $\mathrm{Y}$, et al. Peroral video cholangioscopy to evaluate indeterminate bile duct lesions and preoperative mucosal cancerous extension: A prospective multicenter study. Endoscopy 2013;45:635-42.

13. Nishikawa T, Tsuyuguchi T, Sakai Y, et al. Preoperative assessment of longitudinal extension of cholangiocarcinoma with peroral video-cholangioscopy: A prospective study. Dig Endosc 2014;26:450-7.

14. Nishikawa T, Tsuyuguchi T, Sakai Y, et al. Comparison of the diagnostic accuracy of peroral video-cholangioscopic visual findings and cholangioscopy-guided forceps biopsy findings for indeterminate biliary lesions: A prospective study. Gastrointest Endosc 2013;77:219-26.

15. Kato M, Onoyama T, Takeda Y, et al. Peroral cholangioscopy-guided forceps biopsy and endoscopic scraper for the diagnosis of indeterminate extrahepatic biliary stricture. J Clin Med 2019;8:E873.

16. Hartman DJ, Slivka A, Giusto DA, et al. Tissue yield and diagnostic efficacy of fluoroscopic and cholangioscopic techniques to assess indeterminate biliary strictures. Clin Gastroenterol Hepatol 2012;10:1042-6.

17. Ikeda M, Maetani I, Terada K, et al. Usefulness of endoscopic retrograde biliary biopsy using large-capacity forceps for extrahepatic biliary strictures: A prospective randomized study. Endoscopy 2010;42:837-41.

18. Hijioka S, Hara K, Mizuno N, et al. A novel technique for endoscopic transpapillary "mapping biopsy specimens" of superficial intraductal spread of bile duct carcinoma. Gastrointest Endosc 2014;79:1020-5.

19. Ogawa T, Ito K, Koshita S, et al. Usefulness of cholangioscopic-guided mapping biopsy using SpyGlass DS for preoperative evaluation of extrahepatic cholangiocarcinoma: A pilot study. Endosc Int Open 2018;6:E199-E204.

20. Tsuyuguchi T, Fukuda Y, Saisho H. Peroral cholangioscopy for the diagnosis and treatment of biliary diseases. J Hepatobiliary Pancreat Surg 2006;13:94-9.

21. Nishikawa T, Tsuyuguchi T, Sakai Y, et al. Factors affecting the accuracy of endoscopic transpapillary sampling methods for bile duct cancer. Dig Endosc 2014;26:276-81. 
22. Sugimoto S, Matsubayashi H, Kimura H, et al. Diagnosis of bile duct cancer by bile cytology: usefulness of post-brushing biliary lavage fluid. Endosc Int Open 2015;3:E323-E328.

Cite this article as: Onoyama T, Takeda $\mathrm{Y}$, Kawata S, Kurumi H, Koda H, Yamashita T, Hamamoto W, Sakamoto Y, Matsumoto K, Isomoto H. Adequate tissue acquisition rate of peroral cholangioscopy-guided forceps biopsy. Ann Transl Med 2020;8(17):1073. doi: 10.21037/atm-20-2738
23. Naitoh I, Nakazawa T, Kato A, et al. Predictive factors for positive diagnosis of malignant biliary strictures by transpapillary brush cytology and forceps biopsy. J Dig Dis 2016;17:44-51. 